\title{
CONHECIMENTO TÁTICO DECLARATIVO EM JOGADORES \\ DE FUTEBOL SUB-14 E SUB-15
}

DOI 105902/0102830814693

Data de submissão: 04/08/2014 Data de aceite: 04/11/2014

\author{
Pedro Drumond Moreira \\ Universidade Federal de Minas Gerais \\ pedrodrumondmoreira@hotmail.com \\ Vinicius de Oliveira Viana Soares \\ Universidade Federal de Minas Gerais \\ viniciusovsoares@yahoo.com.br \\ Gibson Moreira Praça \\ Universidade Federal de Minas Gerais \\ gibson_moreira@yahoo.com.br \\ Cristino Julio Alves da Silva Matias \\ Universidade Federal de Minas Gerais \\ crismatias@gmail.com \\ Pablo Juan Greco \\ Universidade Federal de Minas Gerais \\ grecopj@ufmg.br
}

RESUMO: Este artigo comparou o Conhecimento Tático Declarativo (CTD) de jogadores de Futebol das categorias sub-14 e sub-15. Utilizou-se o teste de Mangas, com cenas de vídeo previamente validadas, aplicando-o em 26 atletas do sexo masculino (16 atletas sub-15 e 10 atletas sub-14). Os dados foram analisados através do Median Test. Atletas da categoria sub-15 apresentaram CTD significativamente superior aos atletas sub-14, revelando que o tempo de prática apresenta-se como importante no desenvolvimento do CTD de jogadores de Futebol.

Palavras-chave: Cognição-ação. Jogos Esportivos Coletivos.Futebol 


\section{Introdução}

O futebol é uma modalidade inserida nos denominados Jogos Esportivos Coletivos (JEC), caracterizada por situações de alta variabilidade, imprevisibilidade e aleatoriedade, nas quais duas equipes confrontam-se simultaneamente em um espaço comum, tendo cada uma delas objetivos específicos(GARGANTA, 1997). Para tal, elas devem gerir, em proveito próprio, o tempo e o espaço, realizando-se ações coordenadas de ataque/defesa (GARGANTA, 1997).O jogo oferece um contexto de imprevisibilidade e de incerteza, exigindo a manutenção de estados de alerta para um conjunto diversificado de indicadores(PASSOS et al., 2008)e a obtenção de aptidão nos JEC depende da sobremaneira da capacidade de tomar decisões (WILLIAMS, A M; ERICSSON, 2005). O processo de tomada de decisão emerge do modo como interagem diferentes processos cognitivos como a atenção(Janelle e Hatfield,2008), a antecipação (Tenenbaum,2003) e a memória (DODDS; GRIFFIN; PLACEK, 2001; MATIAS; GRECO, 2010).

Estes processos relacionam-se com o denominado Conhecimento Tático Declarativo - CTD - refere-se às estruturas que podem ser representadas na memória por meio de uma rede de conceitos e suas relações, ou seja, é a capacidade de associação entre os conceitos(ANDERSON; BOTHELL; BYRNE, 2004). Portanto, saber mais significa ter uma rede de ligação entre os conceitos mais ampla, relacionando cada elemento $(\mathrm{CHI}$; GLASSER, 1980).A capacidade tática compreende a interação de processos cognitivos que permitem a tomada de decisão, entendida também como o conjunto de processos psíquicocognitivo-motores determinados para a interação no espaço-tempo e situação (GRECO, P. J., 2006; KANNEKENS; ELFERINK-GEMSER; VISSCHER, 2011; SILVA, M V et al., 2011). A capacidade tática, bem como a qualidade das tomadas de decisão são requisitos para o sucesso esportivo nos JEC(MATIAS; GRECO, 2010), incluindo-se portanto o Futebol. O Conhecimento Tático Declarativo (CTD) é o conhecimento de fatos, isto é, a identificação de estruturas de jogo estabelecidas, identificação de regras de ação, de princípios táticos do jogo, normas do jogo e a posição dos jogadores (CHI; GLASSER, 1980). É caracterizado como informação sobre o modo de executar uma sequência de operações, ou seja, a compreensão e tomada de consciência de como realizar habilidades ou tarefas específicas, (ANDERSON, 2004).Especificamente, o conhecimento tático declarativo (CTD) pode ser 
entendido comoo conhecimento do atleta em saber "o que fazer" em determinado momento do jogo (GARGANTA, 1998). Na literatura, a arquitetura cognitiva do Controle Adaptativo do Pensamento - Racional(ACT-R) formulada por Anderson (2004) propõe uma sequência do conteúdo declarativo para o processual, denominada de proceduralização, no qual o conhecimento declarativo serviria como base para a manifestação processual. Assim, torna-se importante estudar o CTD pelo fato da ação executada pelo praticante no jogo se associar com a forma com que o praticante envolve-se com o contexto do jogo (ARAÚJO, 2009; GRECO, P J, 2007).Com isso, atletas experts nos esportes se destacam dos seus pares no que diz respeito ao desempenho cognitivo e motor, maximizando resultados, por exemplo, no âmbito do conhecimento tático, declarativo e processual, bem como pela elevada taxa de sucesso na execução das técnicas de jogo (FRENCH et al., 1996).

Estudos demonstram que o nível de experiência dos atletas apresenta-se como um aspecto essencial no conhecimento declarativo de praticantes dos JEC, como o estudo de Giacomini, Silva e Greco (2011), que através do protocolo de Mangas (1999) verificou-se que jogadores infantis de Futebol (sub-15) apresentaram CTD superior do que a categoria préinfantil (sub-14). Contudo, atletas Juvenis apresentaram esta melhora em relação ao Infantil, logo não é apenas a experiência que interfere no CTD, resultados que corroboram outros trabalhos da literatura(GIL et al., 2012; MENDES, 1999; TAVARES, 1993; WILLIAMS, A. M.; WARD; SMEETON, 2004) no qual menciona-se que as diferenças podem ocorrer pela propriedade da vivência da modalidade. Em outro trabalho (ABURACHID; SILVA; GRECO, 2013) avaliou-se o nível de conhecimento tático declarativo em jogadores de futebol com faixas etárias de 14-20 anos e observaram-se diferenças significativas entre a faixa etária de 14 anos em comparação as de 15,17 e 20 anos. Porém não houve diferença significativa entre as de 15,17 e 20 anos. Por fim, em um estudo no voleibol, não se observaram diferenças no CTD entre os gêneros (masculino e feminino), mas houve diferenças entre experts (profissionais) e demais escalões (MATIAS; GRECO, 2013).

Observa-se na literatura uma divergência de resultados nos estudos em relação à influência da idade no Conhecimento Tático Declarativo. Além disso, poucos estudos investigaram o CTD no Futebol a partir do paradigma da tomada de decisão nas categorias sub-14 e sub-15, as quais são as primeiras com competições em nível nacional. Desta 
forma, este estudo objetiva comparar o nível de conhecimento tático declarativo (CTD) e o tempo de prática em jogadores, nas categorias sub-15 e sub-14 membros de uma equipe de Futebol.

\section{Materiais e Métodos}

Esse estudo respeitou os procedimentos necessários para garantir o bem estar dos participantes voluntários, recebeu parecer favorável do Comitê de Ética em Pesquisa para o início do projeto, sob o protocolo $\mathrm{n}^{\circ}$. ETIC0218.0.203.000-10. Além disso, todos os responsáveis pelos atletas assinaram o Termo de Consentimento Livre e Esclarecido (TCLE).

A amostra deste estudo foi determinada por julgamento (não probabilística). O critério adotado na eleição dos voluntários descrevia que os indivíduos participantes do estudo deveriam ser atletas que praticavam Futebol em equipes de alto rendimento. A amostra teve ao todo 26 voluntários, composta por atletas inscritos na Federação de Futebol de Minas Gerais. A categoria sub-15 (faixa etária $\leq 15$ anos de idade)contou com a participação de 16 indivíduos e a sub-14 com 10.A média do tempo de prática na modalidade esportiva dos atletas era de $3,5( \pm 1,7)$ anos na categoria sub-15 e 1,5 $( \pm 0,5)$ anos na categoria sub-14. Os atletas realizavam me média sete sessões de treino semanais.

\section{Instrumento}

Para avaliar o CTD dos jogadores foi utilizado o instrumento construído por Mangas (1999). O teste de CTD utiliza-se de medidas de autorrelato. Nesse tipo de teste, as respostas fornecidas pelos sujeitos são utilizadas para medir aspectos do pensamento em relação ao possível comportamento humano no esporte (THOMAS; NELSON; SILVERMAN, 2007). O teste desenvolvido por Mangas (1999) apresenta onze cenas de situações ofensivas de jogos de futebol de alto nível dos principais campeonatos europeus (Espanha, Inglaterra, Itália e Alemanha). As imagens tem duração de 8 a 12 segundos e a ação é paralisada no momento em que o portador da bola deve tomar uma decisão. No teste, após congelar a imagem, se delimitam quatro soluções para cada jogada e não há hierarquia entre as decisões erradas. Giacomini (2007) realizou um estudo no qual se objetivou determinar 
a existência de uma sequência de alternativas de decisão. Os seis peritos (treinadores) consultados elaboraram respostas coincidentes, assim houve uma hierarquia entre as soluções de cada uma das oito jogadas. No presente estudo utilizou-se o instrumento adaptado por Giacomini (2007), considerando-se assim a melhor resposta possível e a hierarquia (de $2^{a}$ a $4^{a}$ ) entre as demais respostas.

\section{Procedimentos de coleta de dados}

$\mathrm{Na}$ aplicação do deste as imagens foram apresentadas aos participantes via projeção em tela, com projetor de multimídia e computador. As cenas da situação de jogo foram paralisadas durante 2 segundos, no momento em que o portador da bola ia decidir "o que fazer". A partir disso, surgiram na tela do computador fotografias, numeradas de 1 a 4 , com as possíveis soluções para as jogadas (figura 1).

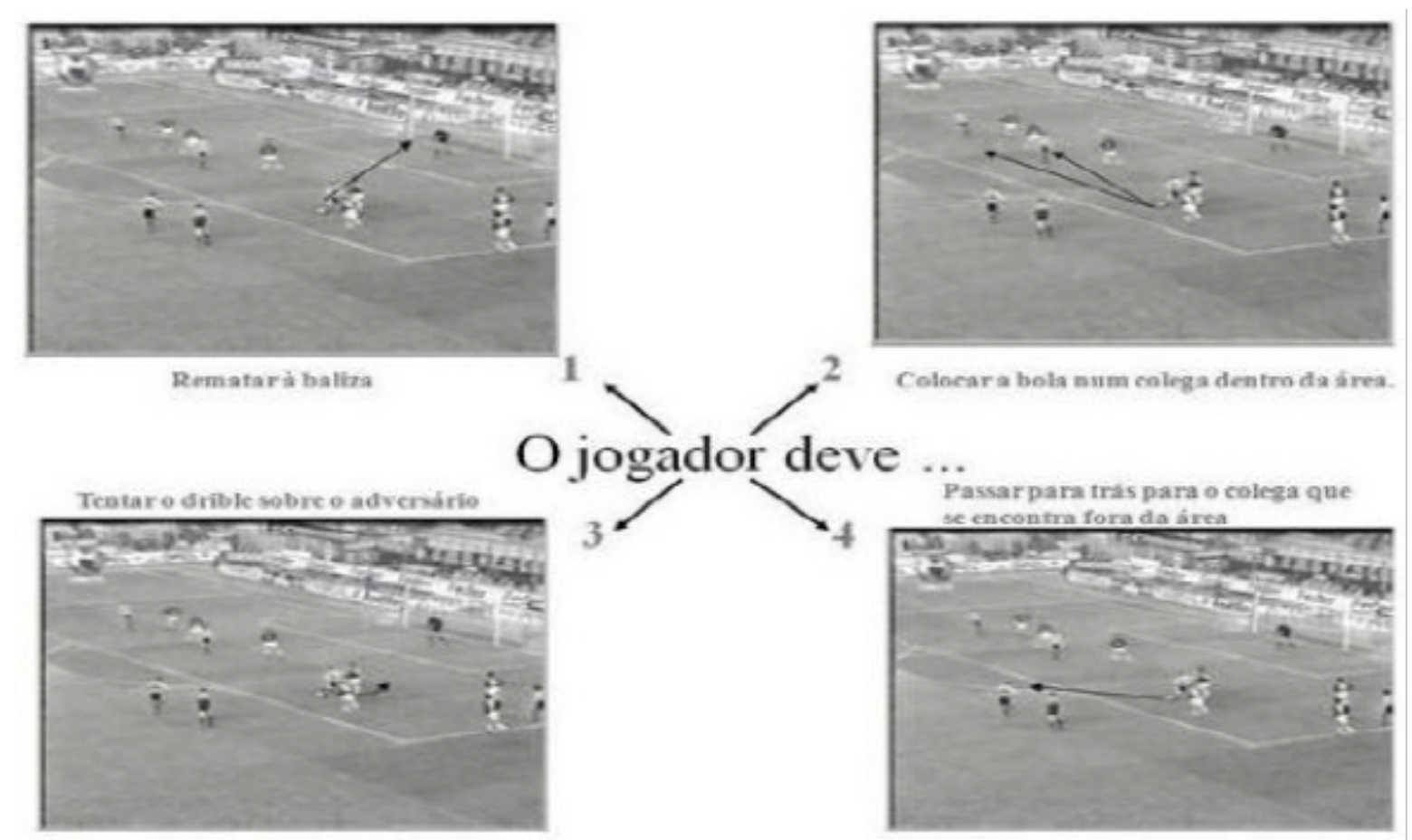

Figura 1. Exemplo da aplicação do teste de CTD (MANGAS, 1999).

O jogador teve o tempo que estimasse necessário para decidir qual seria sua resposta, anotando em uma ficha individual a solução que julgasse mais adequada para a jogada, além de hierarquizar as demais alternativas. 
As instruções iniciais foram ministradas de forma padronizada, sem se modificar o texto proposto por Mangas, de forma a evitar que informações relevantes ou discordantes fossem repassadas para os participantes.

No presente estudo, com base em Giacomini (2007) os seguintes critérios foram utilizados:

a) Melhor solução: $100 \%$ de acerto $=100$ pontos no escore final;

b) $2^{\mathrm{a}}$ melhor solução: $75 \%$ de acerto $=75$ pontos no escore final;

c) $3^{\mathrm{a}}$ melhor solução: $50 \%$ de acerto $=50$ pontos no escore final;

d) Pior solução: $25 \%$ de acerto $=25$ pontos no escore final.

Ou seja, com base nas oito imagens que compõe o teste, a pontuação máxima que pode ser atingida por um atleta é 800 .

Inicialmente realizou-se o teste de normalidade de Shapiro-Wilk, recomendado na literatura para estudos com tamanho amostral pequeno $(n<30)$ A seguir, optou-se pelo Median Test para comparar as medianas do desempenho no teste de CTD entre as categorias. Além disso, realizou-se o teste t para verificar se o tempo de prática dos jogadores se diferenciava significativamente.

\section{Resultados}

A Figura 2 apresenta os resultados descritivos que comparam as categorias sub-15 e sub-14.

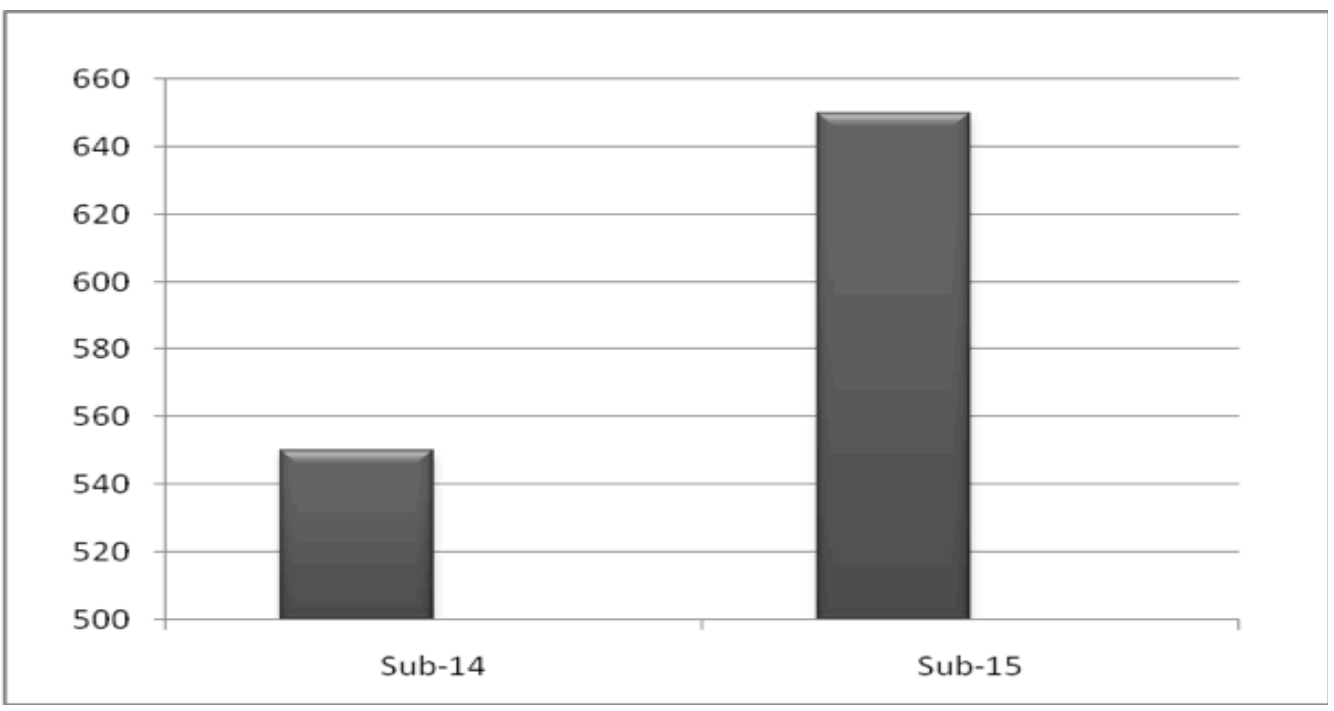

Figura 2. Comparação dos resultados do teste de CTD 
A Figura 2 permite visualizar as diferenças entre as categorias. Nota-se que a categoria sub-15 possui uma pontuação mediana superior à sub-14. A categoria sub-15 apresentou uma mediana de aproximadamente 650 pontos, já a categoria sub-14 uma mediana de 550 pontos. A seguir na tabela 1 é apresentada a comparação do CTD entre as categorias

\begin{tabular}{lcrcc} 
Categoria & Média & Desvio-padrão & Median Test & p valor \\
\hline Pré-infantil & 554,412 & 74,601 & 7,556 & 0,006 \\
Infantil & 655,882 & 64,061 & & \\
\hline
\end{tabular}

Tabela 1: Comparação do CTD entre as categorias

$\mathrm{Na}$ tabela 1 observa-se que houve diferenças significativas entre atletas da categoria sub-14 e categoria sub-15 para a variável CTD $(p=0,006)$. A tabela 2 apresenta os dados referentes à comparação entre o tempo de prática (em anos) nas diferentes categorias.

\begin{tabular}{lcccc} 
Categoria & Média & Desvio-Padrão & $\mathrm{t}$ & $\mathrm{p}$ \\
\hline Sub-14 & 3,560 & 0,631 & 2,720 & 0,012 \\
Sub-15 & 4,238 & 0,610 & & \\
\hline
\end{tabular}

Tabela 2: Comparação do tempo de prática entre as categorias

A partir da tabela 2 observou-se que o tempo de prática entre as categorias foi significativamente diferente. A categoria sub-14 apresentou menor tempo de prática comparativamente à sub-15 $(p=0,012)$.

\section{Discussão}

O presente estudo evidencia uma diferença significativa entre os níveis de CTD, no qual permitiu um maior nível de CTD para a categoria sub-15 $(655,882)$ em frente à categoria sub-14 $(554,412)$. O tempo de prática entre as categorias apresentou-se também estatisticamente diferente $(p=0,012)$, o que pode justificar as diferenças no CTD.

Os resultados desse estudo corroboram com outro estudo na literatura (BRITO; MAÇÃS, 1998) que também comparou o nível de CTD entre categorias (sub-18, sub- 16 e sub-14) e verificou que o nível de CTD foi melhor, em média, da categoria sub-18, em comparação das inferiores. Em adição, corrobora com o estudo de Giacomini (2007) que utilizou o teste de Mangas (1999) e verificou diferenças significativas no CTD entre as categorias Pré- 
infantil (sub-14), infantil (sub-15) e juvenil (sub-17).

Nos estudos de Willians e Davids (1995) menciona-se que o maior nível de CTD das categorias mais experientes dá se também pelo tempo de prática, o que corrobora outros estudos(GIACOMINI; SILVA; GRECO, 2011; MATIAS; GRECO, 2013).Portanto, no presente estudo as diferenças entre as categorias, sub-14 e sub-15, mostradas nos resultados podem ser pelo tempo de prática da modalidade futebol da categoria sub-15. As diferenças entre as categorias pode se dar pelo instrumento utilizado ser capaz de avaliar somente a tomada de decisão, e não os sinais relevantes indicativos a percepção no jogo, que demandaram dos atletas um maior conhecimento sobre as ações do jogo, diminuindo assim, os escores(ABURACHID et al., 2013).

Estudos indicam que os métodos de treinamentos com ênfase no desenvolvimento tático apresentam-se como mais apropriados para o desenvolvimento do conhecimento tático declarativo e processual(FRENCH et al., 1996; GRIFFIN et al., 2001; LIMA; MATIAS; GRECO, 2012; MATIAS; GRECO, 2013; MCPHERSON; THOMAS, 1989; MOREIRA; MATIAS; GRECO, 2013; PEREZ MORALES, 2007). Assim, observa-se que os métodos de ensino situacionais centram-se na tática e permite aos atletas vivenciar situações semelhantes à demanda do jogo real num contexto eminentemente tático (GRECO, P. J.; BENDA, 1998). Assim é ofertado ao atleta uma maior possibilidade de compreensão do jogo, por meio de um processo mais ativo no processo de ensino-aprendizagem-treinamento. Logo, o atleta terá uma maior autonomia em suas decisões. As soluções táticas dos atletas, inerentes as múltiplas situações problemas do jogo, serão cunhadas com inteligência e/ou criatividade (COSTA; NASCIMENTO, 2004; NASCIMENTO et al., 2009; SILVA, M. V.; GRECO, 2009).A partir da construção do saber tático, por meio do ensino-aprendizagem-treinamento situacional, os jogadores estarão em condições de agir "sabendo o que fazer" dentro do jogo.

Pouca atenção tem se dedicado ao desenvolvimento do conhecimento tático no decorrer do período de treinamento, de forma particular, do conhecimento declarativo. Assim o método tradicional, centrado na técnica, ainda é frequentemente utilizado em clubes e escolinhas de esportes. Contrapondo essa concepção, sugere-se que o ensino da técnica seja operacionalizado principalmente através dos contextos do jogo, isto é, de forma situacional (MESQUITA, 2006;2013). 


\section{Conclusão}

A partir dos resultados e discussão relatados acima, a categoria sub-15 apresentou um maior índice de conhecimento declarativo em comparação com a categoria sub-14. A principal razão para esta diferença diz respeito à quantidade de tempo de prática dos atletas, significativamente superior nos atletas da categoria sub-15 $(p=0,012)$. Tais resultados implicam na adequação das atividades de treino às necessidades dos atletas e equipes. Neste âmbito, sugere-se que as intervenções de ensino sejam, concomitantemente, adequadas à realidade dos atletas e suficientes para estimular o estabelecimento de novas redes de relações que amparam o conhecimento.

Salienta-se a necessidade de considerar as limitações do presente aporte. Neste sentido, o desconhecimento acerca do processo de treinamento dos atletas, o tamanho amostral e as características do protocolo de análise devem ser minimizadas com futuras investigações.

Por fim, sugere-se também a investigação acerca de novas ferramentas para acessar o Conhecimento Tático Declarativo, bem como relações estabelecidas com outras áreas de pesquisa. Especificamente, a utilização de equipamentos de rastreamento ocular pode permitir entender o comportamento de busca visual de atletas de diferentes níveis de expertise, e assim entender com mais profundidade as dinâmicas envolvidas no aprendizado de estruturas declarativas de conhecimento ao longo dos anos de prática. 


\section{Referências}

ABURACHID, L. M. C.; SILVA, S. R.; GRECO, P. J. Nível de conhecimento tático de jogadores e avaliação subjetiva dos treinadores de futebol. Revista Brasileira de Futsal e Futebol, v. 5, n. 18, p. 322-330, 2013.

ANDERSON, J. R.; BOTHELL, D.; BYRNE, M. D. An integrated theory of the mind. Psychological Review, v. 111, n. 4, 2004.

ARAÚJO, D. O desenvolvimento da competência tática no desporto: o papel dos constrangimentos no comportamento decisional. Revista Motriz, v. 15, 2009.

BRITO, J.; MAÇÃS, V. A decisão técnico-tática do jogador de futebol: estudo comparativo dos processos percepto-cognitivos inerentes à decisão técnico-tática em sujeitos dos 12 aos 18 anos, federados e não federados em futebol. Horizonte, v. 14, n. 81, 1998.

CHI, M. T. H.; GLASSER, R. The measurement of expertise: analysis of the development of knowledge and skill as a basis for assessing achievement. Educational testing and evaluation. Beverly Hills, 1980.

COSTA, L. C. A.; NASCIMENTO, J. V. O ensino da técnica e da tática: novas abordagens metodológicas. Revista da Educação Física/UEM, v. 15, n. 1, p. 49-56, 2004.

DODDS, P.; GRIFFIN, L.; PLACEK, J. A selected review of the literature on the development of learner's domain-specific knowledge. Journal of Teaching in Physical Education, v. 20, p. 301-313, 2001.

FRENCH, K. E. et al. The Effects of a 3-Week Unit of Tactical, Skill or Combined Tactical and Skill Instruction on Badminton Performance of Ninth-Grade Students. Journal of Teaching in Physical Education, v. 15, n. 4, p. 439-463, 1996.

GARGANTA, J. M. Modelação tática do jogo de futebol: estudo da organização da fase ofensiva em equipas de alto rendimento. Faculdade de Ciênicas do Desporto: Universidade do Porto. Doutorado 1997.

GIACOMINI, D. S.; SILVA, E. G.; GRECO, P. J. Comparação do conhecimento tático declarativo de jogadores de futebol de diferentes categorias e posições. Revista Brasileira de Ciências do Esporte, v. 33, n. 2, 2011.

GIL, A. et al. Analysis of declarative and procedural knowledge in volleyball according to the level of practice and players' age. Perceptual \& Motor Skills, v. 115, n. 2, p. 632-644, 2012. 
GRECO, P. J. Conhecimento tático-técnico: eixo pendular da ação tática (criativa) nos jogos esportivos coletivos. Revista Brasileira de Educação Física e Esporte, v. 20, 2006.

GRECO, P. J. Tomada de decisão nos jogos esportivos coletivos: o conhecimento tático-técnico como eixo de um modelo pendular. Revista Portuguesa de Ciências do Desporto, v. 7, n. Sup., 2007.

GRECO, P. J.; BENDA, R. N. Iniciação Esportiva Universal. Belo Horizonte: Editora UFMG, 1998.

GRIFFIN, L. et al. Middle school students' conceptions of soccer: their solutions to tactical problems. Journal of Teaching in Physical Education, v. 20, n. 4, p. 324-340, 2001.

KANNEKENS, R.; ELFERINK-GEMSER, M. T.; VISSCHER, C. Positioning and deciding: key factors for talent development in soccer. Scandinavian Journal of Medicine \& Science in Sports, v. 21, p. 846-852, 2011.

LIMA, C. O. V.; MATIAS, C. J. A. S.; GRECO, P. J. O conhecimento tático produto de métodos de ensino combinados e aplicados em sequências inversas no voleibol. Revista Brasileira de Educação Física e Esporte, v. 26, n. 1, 2012.

MATIAS, C. J. A. S.; GRECO, P. J. Cognição \& ação nos jogos esportivos coletivos. Ciências \& Cognição, v. 15, n. 1, p. 252-271, 2010.

. O conhecimento tático declarativo dos levantadores campeões de voleibol.

Motriz. Revista de Educação Física. UNESP, v. 19, n. 1, p. 185-194, 2013.

MCPHERSON, S. L.; THOMAS, J. R. Relation of Knowledge and Performance in Boys' Tennis: Age and expertise. JOURNAL OF EXPERIMENTAL CHILD PSYCHOLOGY, v. 48, ก. 2, 1989

MENDES, L. A defesa no processo de preparação desportiva em basquetebol. 1999. (Mestrado). FADE-UP, Universidade do Porto, Portugal.

MESQUITA, I. Ensinar bem para aprender melhor o jogo de voleibol. In: TANI, G.;BENTO, J., et al (Ed.). Pedagogia do Desporto. Rio de Janeiro: Guanabara Koogan, 2006. . Perspectiva construvista da aprendizagem no ensino do jogo In: NASCIMENTO, J. V.;RAMOS, V., et al (Ed.). Jogos Desportivos: formação e investigação. Florianópolis: UDESC, 2013. 
MOREIRA, V. J. P.; MATIAS, C. J. A. S.; GRECO, P. J. A influência dos métodos de ensino-aprendizagem-treinamento no conhecimento tático processual no futsal. Revista Motriz, v. 19, 2013.

NASCIMENTO, J. V. et al. Formação acadêmica e intervenção pedagógica nos esportes. Motriz. Revista de Educação Física. UNESP, v. 15, n. 2, p. 358-366, 2009.

PASSOS, P. et al. Manipulating constraints to train decision making in rugby union. International Journal of Sports Science and Coaching, v. 3, n. 1, p. 125-140, 2008.

PEREZ MORALES, J. C. O processo de ensino-aprendizagem-treinamento no basquetebol: influência no conhecimento tático processual. 2007. (Mestrado). Escola de Educação Física, Fisioterapia e Terapia Ocupcional, Universidade Federal de Minas Gerais, Belo Horizonte.

SILVA, M. V.; GRECO, P. J. A influência dos métodos de ensino-aprendizagemtreinamento no desenvolvimento da inteligência e criatividade tática em atletas de futsal. Revista Brasileira de Educação Física e Esporte, v. 23, 2009.

SILVA, M. V. et al. Estratégia e tática no futsal: uma análise crítica. Caderno de Educação Física, v. 10, 2011.

TAVARES, F. A capacidade de decisão táctica no jogador de basquetebol: estudo comparativo dos processos perceptivo-cognitivos em atletas seniores e cadetes. 1993. (Doutorado). FADE-UP, Universidade do Porto, Portugal.

WILLIAMS, A. M.; ERICSSON, K. A. Perceptual-cognitive expertise in sport: some considerations when applying the expert performance approach. Hum Mov Sci, v. 24, p. 283-307, 2005.

WILLIAMS, A. M.; WARD, P.; SMEETON, N. J. Perceptual and cognitive expertise in sport: implications for skill acquisition and performance enhancement. In: WILLIAMS, A. M. e HODGES, N. J. (Ed.). Skill Acquisition in Sport: Research, Theory and Practice. Londres: Routledge, 2004. 


\title{
Declarative tactical knowledge in under-14 and under-15 soccer players
}

\begin{abstract}
This article compared the Declarative Tactical Knowledge (DTK) of Under-14 and Under-15 soccer players. Mangas' test was used to acces the DTK of 26 soccer players (16 U-15 and $10 \mathrm{U}-10$ ). Data was analysed through the Median Test. U-15 players presented significantly bigger scores in DTK than U-14 players, what indicates the importance of the experience on the DTK development in soccer players.
\end{abstract}

Key-Words: Cognition-Action; Team Sports; Soccer

\section{Conocimiento táctico declarativo de jugadores de fútbol en la sub-14 y sub-15}

RESUMEN: El presente articulo comparó el nivel de conocimiento táctico declarativo (CTD) de jugadores de Fútbol de las categorias sub-14 y sub-15. Se utilizó el test de mangas, que proyecta cenas de juego en video las que fueron previamente validadas. El test se aplicó a una muestra de población de 26 atletas del sexo masculino (16 atletas sub-15 y 10 atletas sub-14). Los datos fueron analizados por medio del test median. Atletas de la categoria sub15 presentaron CTD significativamente superior a los atletas sub-a14, revelando, por lo tanto, que el tiempo de práctica es un factor importante en el desarrollo del CTD en jugadores de Fútbol.

Palabras-clave: Cognición-acción. Juegos Deportivos Colectivos. Fútbol. 